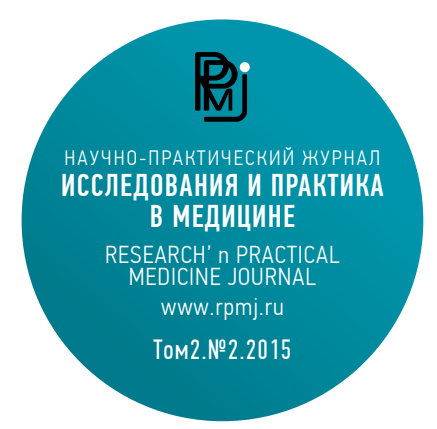

Ключевые слова:

первично операбельный

рак молочной железы,

иммуногистохимия,

орагносохраняющие операции,

комплексная терапия,

комбинированное лечение

Keywords:

first operable cancer breast, immunohystiochemistry,

reconstruction,

organ-preserving operations, complex therapy

DOI: 10.17709/2409-2231-2015-2-2-35-43

\section{ПРОБЛЕМЫ ДИАГНОСТИКИ И ЛЕЧЕНИЯ ПЕРВИЧНО ОПЕРАБЕЛЬНОГО РАКА МОЛОЧНОЙ ЖЕЛЕЗЫ}

Трошенков Е.А. ${ }^{1}$, Костин А.А. ${ }^{2}$

1 МНИОИ им. П.А. Герцена - филиал ФГБУ «НМИРЦ» Минздрава России

125284, Российская Федерация, г. Москва, 2-ой Боткинский проезд, дом 3

2 ФГБУ «НМИРЦ» Минздрава России

249036, Российская Федерация, Калужская область, г. Обнинск, ул. Королёва, д. 4

\section{Резюме}

Рак молочный железы - гетерогенное заболевание, обладающее генотипической и фенотипической вариабельностью. Фенотипическое разнообразие опухолей напрямую связано с генотипическими вариациями, которые можно определить с помощью анализа экспрессии генов при комплексном иммуногистохимическом исследовании. На сегодняшний день существует великое множество методик хирургического лечения - от малоинвазивных до радикальных-, вариабельных по количеству этапов, наличия и объема реконструктивных приемов. На передний план выходят органосохраняющие операции.

\section{PROBLEMS OF DIAGNOSIS AND TREATMENT OF PRIMARY OPERABLE BREAST CANCER}

Troshenkov E.A. ${ }^{1}$, Kostin A.A. ${ }^{2}$

1 P. Hertsen MORI

2-y Botkinskiy proezd, 3, 125284, Moskva, 125284, Russia

${ }^{2}$ NMRRC

Ulitsa Korolyeva, d. 4, Oblinsk, Kaluzskaya Oblast', 249036, Russia

\section{Abstract:}

Cancer breast cancer is a heterogeneous disease with genotypic and phenotypic variability. Phenotypic diversity of tumors is directly related to genotypic variations, which can be determined with analyzing gene expression in a comprehensive immunohistochemical study. Today there are many methods of surgical treatment - from minimally invasive to radical, variable according to the number of stages, the volume of reconstructive techniques. Today organpreserving operations are prefered by surgeons all the world.

$\begin{array}{ll}\text { Список сокращений } \\ \text { БСЛУ } & \text { биопсия сторожевых лимфатических узлов } \\ \text { ГР } & \text { рецепторы гормонов } \\ \text { ДПлУ } & \text { диссекция подмышечных лимфатических узлов } \\ \text { ИГХ } & \text { иммуногистохимия } \\ \text { ИМИ } & \text { индекс митотической активности } \\ \text { МРТ } & \text { магнитно -резонансная томография } \\ \text { НАТ } & \text { неоадъювантная терапия } \\ \text { ОСО } & \text { органосохраняющие операции } \\ \text { ОСХ } & \text { органосохраняющая хирургия } \\ \text { ОТ - ПцР } & \text { обратно-транскриптазная полимеразная цепная реакция } \\ \text { ППО } & \text { полный патологический ответ } \\ \text { ПР } & \text { рецепторы прогестерона } \\ \text { РМЖ } & \text { рак молочной железы } \\ \text { САК } & \text { сосково-ареолярный комплекс } \\ \text { УзИ } & \text { ульразвуковое исследование } \\ \text { ШР } & \text { шкала рецидивирования } \\ \text { ЭР } & \text { рецепторы эстрогена }\end{array}$




\section{Введение}

Первично операбельный рак молочной железы условное понятие, выделенное специально для клинической практики хирургов-онкологов. Первично операбельный рак характеризуют в зависимости от стадии заболевания по системе TNM: к нему относят РМЖ I- IIB стадии при T1 или T2, N0 или N1 при отсутствии отдаленных метастазов (М0). Данное определение позволяет на дооперационном периоде спланировать объем хирургического и комплексного лечения с учетом современных подходов к проблеме РМЖ, однако оно основано лишь на клинико-патологической классификации без учета внутреннего деления РМЖ на подтипы и подгруппы. За рубежом существует аналогичное первично операбельному раку молочной железы понятие «раннего рака молочной железы», основанное не только на классификации TNM, но и на результатах иммуногистохимического исследования и других современных лабораторных методов исследования. Такой подход позволяет более дифференцировано подходить к выбору хирургических техник и делает прогноз лечения более предсказуемым [1-6].

\section{Основная часть}

Рак молочный железы- гетерогенное заболевание, обладающее генотипической и фенотипической вариабельностью $[7,8]$.

Фенотипическое разнообразие опухолей напрямую связано с генотипическими вариациями, которые можно определить с помощью анализа экспрессии генов. Каждый подтип основан на “внутреннем» списке генов, что отражается на клиническом проявлении подтипа опухоли и прогнозе заболевания [9]. Следует отметить, что в исследованиях, в которых первоначально определяли внутренние подтипы генов, основанные на измерении матричной РНК, была выявлена сегрегация по эстрогеновым рецепторам (ER) до Her2, что позволило предположить важность гормонального статуса как определяющего фактора при раке молочной железы и разделить все случаи заболевания в зависимости от ЭР на две основные группы: ЭР-позитивные (люминальные подтипы А и В) и ЭР-негативный подтипы (нормальные, Her2-обогащенные, базальные и клаудин-низкие (claudin-low) [10, 5, 11]. Люминальные ЭР-позитивные опухоли характеризуются относительно высокой экспрессией многих генов, также как и у нормальных просветных эпителиальных клеток. Уровни экспрессии и пролиферации являются наиболее заметными различиями между люминальными А и Б подтипами РМЖ. Люминальный подтип А связан с наивысшей экспрессией генов, характерной для ЭР кластера, и низкой экспрессией маркеров пролиферации. Люминальный подтип В характеризуется умеренно низкой экспрессией ЭР - связанных генов, переменной экспрессией HER2-ассоциированных генов, более высокой экспрессией маркеров пролиферации и умеренной экспрессией некоторых генов, общих с базальным подтипом опухолей. Предполагают, что люминальный подтип опухоли В является более разнородным, чем подтип А. В целом, все ЭР-негативные подтипы обладают высокой пролиферацией совместно с HER2-acсоциированными генами, что играет важную роль в сегрегации подтипов опухолей $[12,13,14,15]$.
В клинической практике наиболее часто выделяют 4 главных подтипа рака молочной железы, требующих лечения: эстрогеновые рецепторы есть (ЭР -позитивные) и отсутствует человеческий эпидермальный фактор роста -2 , низкая или промежуточная степень дифференцировки; ЭР- позитивные и HER2-негативные с высокой степенью дифференцировки клеток; HER2-позитивные и трипл-негативные опухоли молочной железы (ЭР, рецепторы прогестерона, HER2-отсутствуют).

Более того, недавние сравнительное исследование четырех основных биомаркеров при ИГХ РМЖ- ЭР, ПР, her2 и Ki-67 (ИГХ 4) показало, что полученная прогностическая информация может быть, по крайней мере, эквивалентна шкале рецидива заболевания при оценке генетического здоровья [16].

Последние предложенные рекомендации по выбору адъювантного системного лечения в специфических популяциях людей основывались на определении внутренних биологических подтипов с разным ответом на системную и местную терапию $[17,18]$.

\section{Диагностика}

Традиционные биомаркеры включают в себя клиникопатологические характеристики, которые широко используют в клинической практике как прогностические биомаркеры: размер опухоли, состояние лимфатических узлов, наличие рецепторов эстрогена и прогестерона, человеческого эпидермального фактора роста - 2; степень дифференцировки клеток опухоли; индекс пролиферации [19].

К инструментальным методам исследования, преимущественно применяемых при диагностике РМЖ относят: маммографию, ультразвуковое исследование молочной железы и зон регионарного метастазирования, печени и органов малого таза, радионуклидное исследование костей скелета, рентгенографию органов грудной клетки, МРТ молочных желез, тонкоигольную биопсию с гистологическим исследованием полученных образцов ткани [20].

G. Cancello (2013) в своем исследовании у всех пациентов диагностировал инвазивный РМЖ, что было подтверждено гистологически после проведения кор-биосии под ультразвуковым наведением. По результатам магнитнорезонансной томографии (МРТ) опухоли были классифицированы как уни- или мультифокальные. При отсутствии подозрений на поражение подмышечных лимфатических узлов, а также в случае NO PMЖ, перед проведением неоадъювантной терапии (НАТ) проводили биопсию сторожевых лимфатических узлов (БСлУ) [6].

Однако, в подавляющем большинстве случаев члены экспертной группы 13-й Международной конференции по раку молочной железы St Gallen (2013) посчитали, что магнитно-резонансная томография не должна быть рутинным методом для диагностики впервые выявляемого рака молочной железы [1].

A. Bleyer и соавт. (2012) использовали метод динамического наблюдения за пациентами, изучали эпидемиологию РМЖ и анализировали конечные результаты с 1976 по 2008 гг. с целью изучения возможной тенденции для заболеваемости ранним раком молочной железы (протоковая карцинома in situ, локализованный очаг) и поздними стадиями рака молочной железы (поражения 
регионарных структур и наличие отдаленных очагов поражения) среди женщин 40 лет и старше [21].

Внедрение маммографического скрининга в США было связано с удвоением числа случаев обнаружения ранних стадий рака молочной железы, которые выявляли ежегодно, от 112 до 234 случаев на 100000 женщин - абсолютный прирост составил 122 случая на 100000 женщин. Одновременно, частоты выявления пациенток с поздней стадией рака снизилась на 8\%, с 102 до 94 случаев на 100000 женщин - абсолютное снижение составило 8 случаев на 100000 женщин. После исключения промежуточных значений для частоты заболеваемости, связанной с проведением гормон-заместительной терапии, и корректировки с учетом тенденций в заболеваемости раком молочной железы среди женщин моложе 40 лет, авторы подсчитали, что рак молочной железы был ошибочно диагностирован (т. е. опухоли выявлены по данным скрининга, однако никакой клинической симптоматики не отмечали на протяжении всего периода наблюдения) у 1,3 миллиона жительниц США за последние 30 лет.

Патологоанатомическое исследование с применением радиоактивного йода с ядром 125 проводят согласно стандартам процедуры, включая удаление ядерных зерен. В последующем их возвращают в отделение ядерной медицины для дальнейшей переработки радиоактивных материалов согласно голландским правилам по ядерной энергии [22]. Полный патологический ответ (ППО) определяется микроскопически как отсутствие остаточных опухолевых клеток в резецированном образце. Модифицированная шкала Bloom- Richardson [23] и индекс митотической активности (ИМА) не могут быть оценены в случае ППО, т.к. кор-биопсию, на которой он основан, не проводят в рутинном порядке.

В нескольких обзорных статьях были рассмотрены потенциальные возможности использования традиционных биомаркеров и их недостатки $[24,19,25]$.

Другим важным источником трудностей является определение и оценка гормональных рецепторов (ГР) и позитивности her2. Последние сообщения свидетельствуют о данных трудностях, показывая, что результаты использования обратной транскриптазной полимеразной цепной реакции для оценки her2 не вполне совпадают с результатами стандартных методик, таких как флуоресцентная гибридизация in situ или иммуногистохимия. Кроме того, наблюдается большая вариабельность в использовании пороговых значений для определения ЭР и ПР позитивности и в определении подтипа РМЖ (например, включение (или нет) статус рецепторов прогестерона в определение гормон-позитивного рака молочной железы) $[26,27,28,29,30]$.

\section{Новое в диагностике}

Прогресс в понимании рака молочной железы на молекулярном уровне и разработка сверхсовременных средств диагностики обусловила создание нескольких мультигенных методов анализа, которые помогают в определении отдельных подтипов опухолей. К доступным мультигенным анализам, используемым в клинической практике относят: Oncotype DX и MammaPrint. Oncotype DX-это 21-генная обратно- транскриптазная по- лимеразная цепная реакция (ОТ-ПЦР) на основе анализа 16 генов, связанных с РМЖ, участвующих в пролиферации, инвазии и her2/ЭР сигнализации, а также 5 референсных генов, полученных из первоначальной выборки 250 генов-кандидатов, опубликованных в источниках литературы и геномных базах данных. Исследование проводится на залитой в парафин опухолевой ткани и результат формируется в виде шкалы рецидива (ШР) в диапазоне от 0 до 100 и делится на 3 группы риска: низкий (ШР<18), промежуточный (ШР= 18-30) и высокий (ШР>31). ШР предоставляет информацию о прогнозе РМЖ вне зависимости от традиционных клиникопатологических факторов заболевания. Прогностическая полезность этого теста была подтверждена в большом когортном исследовании из 668 больных NO раком молочной железы, получавших тамоксифен в NSABP B-14 исследовании, которое показало, что 10-летний отдаленный результат в отношение рецидива РМЖ для низкой, промежуточной и высокой ШР составил 6,8\%, 14,3\% и 30.5\% соответственно [31]. В рекомендациях NCCN по лечению рака молочной железы тест Oncotype DX относят к категории рекомендаций 2 В для оценки вероятности рецидива и эффективности химиотерапии при ЭР- позитивном NO раке молочной железы и величине опухолевого очага более 0,5 cм [32]. Прогностическая значимость промежуточной ШР остается неясной и оценивается в перспективе в TAILORx исследовании (Исследование с назначением индивидуализированного варианта лечения; ClinicalTrials.gov идентификатор: NCT00310180), в котором пациентам с ШР от 11 до 25 проводили химиотерапию совместно с эндокринной терапией и моно- эндокринную терапию. Мультицентровое проспективное исследование показало, что результаты Oncotype DX в исследованиях значительно повлияли на решение выбора метода лечения.

MammaPrint является микрочипом, совершающим мультигенный анализ, и предназначен для оценки риска у пациентов с NO раком молочной железы независимо от ЭР- статуса. 70-генная сигнатура состоит из генов, участвующих в пролиферации, инвазии, метастазирования и ангиогенезе. В начале 2012 года, MammaPrint стала доступна для тестирования залитых парафином образцов ткани, что упростило его клиническое применение. MammaPrint тест был тщательно проверен в ретроспективных исследованиях и в настоящее время проходит оценку в проспективном MINDACT (Микрочипы при NO могут помочь избежать химиотерапии; ClinicalTrials. gov идентификатор: NCT00433589), в котором эффект адъювантной терапии у пациентах с NO раком молочной железы оценивается с помощью стандартных клиникопрогностических факторов на основе Adjuvant! Online и MammaPrint. Основываясь на последних результатах исследования NO пациентов в MINDACT были внесены изменения, чтобы включить пациентов с 1 до 3 пораженными лимфатическими узлами [33]. Пациенты с низким риском по результатам обоих тестов не будут получать химиотерапию; пациенты в группе высокого риска по обоим тестам будут получать химиотерапию; пациентам расхождением оценки риска по тестам будет рандомизировано назначена или не назначена химиотерапия [19]. 


\section{Лечение}

Процесс снижения объема хирургического лечения РМЖ на начальных стадиях заболевания начал активно развиваться еще в 70-х годах прошлого столетия. На сегодняшний день существует великое множество методик хирургического лечения - от малоинвазивных до радикальных-, вариабельных по количеству этапов, наличия и объема реконструктивных приемов. На передний план выходят органосохраняющие операции (ОСО). Показанием к их проведению в РФ считают: опухоль до 3-4 см, соотношение размера опухоли и молочной железы, позволяющее выполнить радикальное вмешательство, моноцентричный рост, отсутствие данных о наличии регионарного и отдаленного метастазирования. Следует учитывать, что при инвазивном дольковом раке по сравнению с инфильтративным протоковым раком органосохраняющее лечение сопровождается большим процентом местного рецидивирования, наиболее благоприятными гистологическими формами для проведения органосохраняющего лечения являются некоторые редкие формы рака молочной железы (медуллярный, тубулярный). Желание пациентки сохранить молочную железу - важнейший фактор при органосохраняющем лечении [20].

Проведенные исследования продемонстрировали онкологическую безопасность подкожной мастэктомий с сохранением САК при тщательном отборе пациентов к данному типу оперативного вмешательства. Исходя из вышеизложенного, можно предположить, что подкожная мастэктомия с сохранением САК или без него с одномоментной реконструкцией молочной железы наиболее удовлетворяет как принципам онкологической безопасности, так и создает благоприятные условия для различных вариантов реконструкции молочной железы и облегчает ее. Однако требуется более длительный срок наблюдения за данной группой больных. Одним из наиболее грозных осложнений подкожной мастэктомии с сохранением САК является некроз соска и/или ареолы, что ведет за собой потерю установленного импланта. Процент данного вида осложнений колеблется от 2 до 20\%, более часто наблюдается в группе больных при проведении лучевой терапии [34, 35, 36]. В то время как радикальная мастэктомия с/без одномоментной реконструкцией продолжает оставаться стандартом лечения, подкожная мастэктомия с сохранением САК является альтернативным вариантом хирургического лечения, улучшающая качество жизни пациентов и обеспечивающая сравнимый с радикальными мастэктомиями процент местного рецидивирования. Преимущество органосохраняющего лечения перед радикальными мастэктомиями по сохранению внешнего облика и качества жизни пациентов ни у кого не вызывает сомнения, однако риск возникновения местного рецидива от 8,8 до $15 \%$ останавливает большинство онкологов от широкого внедрения данного вида оперативного вмешательства в клиническую практику. Дальнейшая реконструкция молочной железы после возникновения местного рецидива после органосохраняющего лечения весьма затруднительна из-за проведенных ранее курсов лучевой терапии. Реконструкция молочной железы уменьшает психологическую травму, связанную с маст- эктомией. Условно все реконструктивные операции можно разделить на три вида: с использованием собственных тканей, тканевых экспандеров и эндопротезов и их различная комбинация. Восстановление косметического дефекта после удаления органа - это самый действенный способ психотерапии, однако, осуществляется он только по осознанному желанию самой пациентки. Роль врача в данном случае состоит в предоставлении полноценной информации о методах, результатах и возможных осложнениях реконструктивной хирургии, а также в оценке возможности и сроков выполнения оперативного вмешательства с точки зрения онколога [20].

На 13-й Международной конференции по раку молочной железы St Gallen (2013) группа экспертов рассмотрела и одобрила существенно новые данные по аспектам местного и регионального лечения при раннем раке молочной железы, сделав акцент о необходимости проведения менее обширных операций с переходом на область подмышечной впадины и сокращение сроков лучевой терапии [1].

Недавние исследования по применению местной терапии при раннем РМЖ подтвердили возможность сохранения существующей тенденции к проведению менее обширных процедур. Таким образом, диссекцию тканей и анатомических структур в области подмышечной впадины можно избежать без вреда для пациенток с микрометастазами в сторожевых лимфатических узлах [37] и больных, перенесших органосохраняющие операции на молочной железе и полное облучение молочной железы при наличие не более двух макроскопически пораженных сигнальных лимфатических узлов [38].

На Панели Международной конференции по раку молочной железы St Gallen (2013) эксперты утвердили небольшее число абсолютных противопоказаний к проведению органосохраняющих операций. К одному из таких абсолютных противопоказаний были отнесены случаи краевой инвазии клетками карциономы или внутрипротоковой карциномы после повторной резекции. Минимальным отступом от границы предполагаемого хирургического вмешательства три четверти группы экспертов и большинство других специалистов сочли неокрашенные ткани за пределами патологического очага (т.е. область, не пораженная опухолью) шириной минимум 1 мм. Члены группы были практически единодушны в том, что органосохраняющие операции на МЖ не следует проводить, если существует необходимость проведения в послеоперационном периоде лучевой терапии [1]

Проведение операции с сохранением САК считается целесообразным при условии отсутствия поблизости от соска очага поражения опухолевым процессом.

Эксперты проблемной комиссии 13-й Международной конференции по раку молочной железы St Gallen (2013) посчитали возможным избегать проведения подмышечной лимфодиссекции у пациенток с поражением одного или двух сторожевых лимфатических узлов после органосохраняющих операций, когда планируется полная лучевая терапия всей грудной клетки и Мж. Группа экспертов разделилась во мнении практически поровну в вопросе применения данной рекомендации в случае проведения мастэктомии с последующей лучевой терапией, но были практически единодушны в необходимости 
подмышечной диссекции, если лучевая терапия не была запланирована. Эксперты также посчитали, что подмышечную диссекцию следует проводить в случае поражения трех и более сторожевых лимфатических узлов или их вовлечение в патологический процесс было установлено до хирургического вмешательства и подтверждено по результатам биопсии.

Радикальные резекции по данным голландских онкологических руководств определяют как метод лечения рака молочной железы любой стадии и подтипа. Данную операцию проводят в пределах здоровых тканей при отсутствии опухолевых клеток на границе резекции. Вовлечение в опухолевый процесс тканей на границе патологического очага считают зону $\leq 4$ мм, отступы от окрашенного края резекции. Экстенсивное поражение границ опухоли считают увеличение зоны более 4 мм [4].

Лампэктомия (эктомия опухоли с использование онкопластических техник по показаниям) совместно с диссекцией подмышечных лимфатических узлов (ДПлу) в случае первично диагностирования поражения подмышечных лимфатических узлов проводят через 4-6 недель после завершения НАТ. Ручной гамма- зонд (Europrobe, EuroMedical Instrument, le Chesnay, Франция) применяют интраоперационно, устанавливая над передней поверхностью молочной железы с целью навигации и обеспечения получения образцов тканей при оптимальной резекции. После резекции и зонд, и полученные рентгенологические снимки используют для нахождения введенных радиоактивных частиц І-125 у пациентов. В послеоперационном периоде применения радиоактивного йода не привело к развитию каких- либо осложнений [4].

Полная резекция опухоли в пределах интактных тканей имеет первостепенное значение в органосохраняющей хирургии (OCX), т.к. позволяет предотвратить развитие местных рецидивов, которые связаны с прогностически неблагоприятным исходом заболевания [39]. Это согласуется с отчетами сравнения обычных рекомендованных методов определения границы резекции и техники с применением радиоактивного изотопа йода -125 семян локализации при ранних стадиях рака молочной железы, что позволило улучшить результат с $11 \%$ до 27\% [40]. Более того, по данным литературы, хирурги предпочитают метод определения локализации радиоактивных изотопов йода, т. к. он позволяет сократить время операции и улучшить интраоперационную навигацию [41].

По словам датского руководства по контролю качества хирургического лечения рака молочной железы, иррадикальную резекцию на первом этапе лечения раннего инвазивного рака молочной железы следует проводить <20\% случаев ОСО [42]. В целях обеспечения успеха полной резекции, применяют несколько локальных вспомогательных техник, включая гид-проводник, радиоуправляемое оккультное определение локализации поражения (ROLL) и предоперационное УЗИ, для установления частоты поражения области границы резекции, успех которых порой достигает более 20\% [43]. При органосохраняющих операциях после проведения НАТ, возможно повышение частоты поражения краевой зоны резекции, что связано с первоначально большими размерами опухоли, особен- но в случае ограниченного ответа на проведенную НАТ или мультифокального РМЖ [4].

Поиск более точных методик локализации, разделяющие пораженные опухолью и нормальные ткани интраоперационно, до сих пор является интересным объектом для исследования. Ближняя инфракрасная флуоресценция является перспективным методом для хирургического лечение рака молочной железы в будущем [44] (Pleijhuis RG, 2011). До времени ее широкого внедрения определение локализации зоны опухоли с помощью маркеров, которые могут точно установить области, подлежащие резекции, остается оптимальным для хирургии Мж. Методы локализации основаны на введении радиоактивных частиц Nanocolloid, содержащих жидкости, диффундирующие в ткани молочной железы, тем самым ограничивая объем прецизионной резекции, особенно при мультифокальном заболевании [45] (Espinosa-Bravo M, 2011). Последние нельзя проследить, что требует вторичной локализации с проводником. Многоцентровой ретроспективный анализ проведенных различными хирургами в течение 4 лет ОСО в течение 4-летнего периода показало преимущество УЗИ- контролируемой хирургии непальпируемых опухолей молочной железы и вовлечение в процесс краевой зоны резекции только в 4\% по сравнению с $21 \%$ после применения ручного проводника и $25 \%$ после ROLL техники. Дальнейшую оценку этих методов в настоящее время проводят в проспективных рандомизированных контролируемых исследованиях [47].

Результаты исследования Weissenbacher и соавт. в поддержку радикальной мастэктомии при мультифокальных опухолей, как предполагали авторы, должны быть связаны с повышенным риском вовлечения в процесс краевой зоны резекции и повышением частоты развития местных рецидивов после органосохраняющих операций [47]. Они сообщили о том, что мультифокальность или мультицентричность опухоли выступали как независимый фактор риска развития местных рецидивов у 576 пациентов после ОСО, однако, авторы не обнаружили различий между ними. Это противоречит другим исследованиям, в которых не обнаружили связи между мультифокальностью РМЖ и ростом частоты развития местных рецидивов после проведения ОСО [48]. Следовательно, необходимо тщательно оценивать пациентов на предмет возможности проведения ОСО в зависимости от фокальности опухоли, которая может быть одним из факторов риска. Подтверждение наличия дополнительных патологических очагов вместе с характеристикой самой опухоли молочной железы по сравнению с объемным соотношением является важным элементом диагностики на этапе принятия решения о возможности проведения ОСО. Последующее точное определение локализации всех подтвержденных очагов, является визитной карточкой успешности ОСО. Yerushalmi и соавт. сообщили о частоте развития местных рецидивов РМЖ, которая была сопоставима для пациентов с мультифокальными и монофокальными опухолями [49].

Выделенные подтипы РМЖ характеризуются различными эпидемиологическими факторами риска, генетическим происхождением и отличаются ответом на системную и местную терапию. Клиницистам, занимающимся лечением пациентов, больных РМЖ даже 
первично операбельных, следует до выбора метода лечения точно определить подтип опухоли. Однако определение подтипа опухоли с помощью традиционных биомаркеров такой гетерогенной группы опухолей и дальнейшее внутреннее деление опухоли на подгруппы с целью подбора оптимальной таргетной терапии имеет широкий диапазон. Особенно, это касается люминал В подтипа РМЖ при HER2-негативности опухоли -до сих пор нет единого мнения о необходимости включения химиотерапии в схему лечения.

Гормональная терапия является всего лишь частью лечебной схемы при люминал В подтипе РМЖ. Проведение химиотерапии также показано большинству пациенток с люминал В подтипом опухоли в сочетании с трастузумабом при HER2-позитивном РМЖ [1].

В ряде исследований, гиперэкспрессию her2 связали с резистентностью к эндокринной (гормональной) терапии [50], и схожую ситуацию наблюдали для ЭР, который обозначили как метод таргетной терапии her2 - подтипах опухолей $[51,52]$. Кроме того, было показано, что тамоксифен ведет себя как агонист эстрогена в клетках рака молочной железы, в которых есть гиперэкспрессия генов her2 и AIB1 [51]. Wang и соавт. показали, что после лечения лапатинибом и трастузумабом, уровень ЭР и продуктов их переработки вырос в четырех из пяти ЭР-позитивных/her2-позитивных клеточных линиях. Кроме того, при блокировании her2 с помощью лапатиниба и трастузумаба, приобретение резистентности требует активации пути ЭР посредством вовлечения белков семейства $\mathrm{Bcl} 2$ [30].

Решения в отношении проведения адъювантной химиотерапии у пациентов с ЭР-позитивном, her2-негативном, NO PMЖ традиционно опирались на клинико-патологические характеристики заболевания. Однако, молекулярная неоднородность и сложный комплексный геном опухоли требуют более совершенных подходов к данной проблеме. Было разработано несколько мультигенных аналитических процедур для прогнозирования риска рецидива РМЖ и смерти, оценки эффективности лечения в конкретной популяции людей. Онкологи все чаще сталкиваются с необходимостью включения различных сложных геномных биомаркеров РМж наряду с традиционными в диагностический процесс. На сегодняшний день NCCN руководство в клинической онкологической практике при раке молочной железы полезно лишь тем, что дает общее направление в выборе лечения, однако остаются неопределенными данных в отношение частных клинических ситуаций [19].

В двух больших рандомизированных исследованиях оценивали эффективность ингибиторов ароматазы, летрозола (BIG 1-98 исследование) и анастрозола (АTAC исследование), по сравнению с тамоксифеном, назначенным в качестве адъювантной терапии больных ранним раком молочной железы. B Big 1-98 исследовании, пациенты, получавшие летрозол имели лучший результат, чем те, которых проходили лечение с тамоксифеном вне зависимости от ПР-статуса [54]. Последующий анализ в АТАС исследовании показал, что количественное выражение экспрессии генов ЭР, ПР и her2-статус, не позволило выявить пациентов со значительным различием в достигнутом результате при лечении анастрозолом или тамоксифеном [25, 6].
Оказалось, что у пациентов с ЭР-положительными и ЭР-отрицательными возможно получить хороший лечебный результат от адъювантной полихимиотерапии, однако абсолютная величина эффекта лечения может варьировать в различных подгруппах: совместное групповое исследование раннего операбельного РМЖ показало, что, у женщин в возрасте до 50 лет с ЭР-позитивными опухолями 5-летний абсолютный риск рецидивирования снижался на 8\%, в то время, как у пациенток с ЭР-негативным статусом 5-летний абсолютный риск рецидивирования снижался на 13\%. При her2-позитивном РМЖ в каждом исследовании адъювантной терапии с трастузумабом был отмечен схожий эффект в отношение определяемого снижения относительного риска при добавлении трастузумаба в обеих подгруппах - ER-позитивной и ЭР- негативной и $[55,56,30]$.

Для гормон-рецептор-позитивного рака молочной железы ранней стадии риск рецидива и потенциальный эффект химиотерапии должны быть сбалансированы с потенциальным риском неблагоприятного исхода и возможностью избыточного лечения при отсутствии хорошего результата при применении химиотерапии. Также считают, что вовлечение в процесс лимфатических узлов на стороне поражения не означает автоматического введения в схему лечения химиопрепаратов [1]. Тест Oncotype DX продемонстрировал высокую прогностическую значимость, выходящую за рамки возможностей традиционных биомаркеров при NO и N+ гормон-рецептор-позитивном РМЖ [31, 57]. Недавний мета-анализ [58] показал, что результаты лечения для NO - ЭР-позитивном РМЖ в странах с различными терапевтическими традициями вариабельны в пределах $935 \%$ случаев.

\section{Заключение}

Рак молочной железы представляет собой фенотипическое и генотипическое сложное онкологическое заболевания, при котором клиническая симптоматика, результаты лечения и прогноз зависят от подтипа и подгруппы опухоли, определяемых на основании комплексных методик с привлечением инструментальных методик, традиционных маркеров, постоянно развивающейся иммуногистохимии, основных генетических факторов и инновационных методик на основании мультигенного анализа и применения нанотехнологий. Методы лечения также прошли длительный путь от сложных схем комбинированного, комплексного лечения до сокращения его объема до минимальных - на передний план вышла монотерапия таргетного характера. Однако понимание первично операбельного рака молочной железы разнится у специалистов в РФ и за рубежом, отличаются подходы к диагностике и лечению- до их пор в России практикуют радикальные операции при тех стадиях и подтипах РМЖ, при которых за границей с успехом применяют органосохраняющие операции в сочетании с одномоментной реконструкцией молочной железы при необходимости. Все это послужило основанием для проведения нашей работы. 
Список литературы / References:

1. Goldhirsch A., Winer E.P., Coates A.S., Gelber R.D. Panel members ${ }^{\dagger}$ Personalizing the treatment of women with early breast cancer: highlights of the St Gallen International Expert Consensus on the Primary Therapy of Early Breast Cancer. Annals of Oncology. 2013; 24.(9): 2206-2223.

2. Park S., Koo J., Park H.S., et al. Expression of androgen receptors in primary breast cancer. Ann Oncol. 2010;.21: 488-492.

3. Park S., Park H.S., Kim S.I. The impact of a focally positive resection margin on the local control in patients treated with breast-conserving therapy. J Clin Oncol.2011; 5:.600-608.

4. Gobardhan P.D., de Wall L.L., van der Laan L., ten Tije A.J. The role of radioactive iodine-125 seed localization in breastconserving therapy following neoadjuvant chemotherapy. Ann Oncol. 2013; 24(3): 668-673.

5. Prat A., Perou C.M. Deconstructing the molecular portraits of breast cancer. Mol Oncol. 2010; 5: 5-23.

6. Cancello G. Progesterone receptor loss identifies Luminal B breast cancer subgroups at higher risk of relapse. Annals of Oncology. 2013; 24: 661-668

7. Oh D.S., Troester M.A., Usary J., et al. Estrogen-regulated genes predict survival in hormone receptor-positive breast cancers. J Clin Oncol. 2006; 24: 1656-1664.

8. Rouzier R., Perou C.M., Symmans W.F., et al. Breast cancer molecular subtypes respond differently to preoperative chemotherapy. Clin Cancer Res. 2005; 11: 5678-5685.

9. Sorlie T., Tibshirani R., Parker J., et al. Repeated observation of breast tumor subtypes in independent gene expression data sets. Proc Natl Acad Sci USA. 2003; 100: 8418-8423.

10. Hennessy B.T., Gonzalez-Angulo A.M., Stemke-Hale K., et al. Characterization of a naturally occurring breast cancer subset enriched in epithelial-to-mesenchymal transition and stem cell characteristics. Cancer Res. 2009; 69: 4116-4124.

11. Prat A., Parker J.S., Karginova O., et al. Phenotypic and molecular characterization of the claudin-low intrinsic subtype of breast cancer. Breast Cancer Res. 2010; 12: R68.

12. Marcom P.K., Isaacs C., Harris L., et al. The combination of letrozole and trastuzumab as first or second-line biological therapy produces durable responses in a subset of HER2 positive and ER positive advanced breast cancers. Breast Cancer Res Treat. 2007; 102: 43-49.

13. Cui X., Schiff R., Arpino G., et al.. Biology of progesterone receptor loss in breast cancer and its implications for endocrine therapy. J Clin Oncol. 2005;.23: 7721-7735.

14. Rakha E.A., El-Sayed M.E., Green A.R., et al.. Biologic and clinical characteristics of breast cancer with single hormone receptor positive phenotype. J Clin Oncol. 2007; 25: 4772-4778.

15. Creighton C.J., Kent Osborne C., van de Vijver M.J., et al.. Molecular profiles of progesterone receptor loss in human breast tumors. Breast Cancer Res Treat. 2009; 114: 287-299.

16. Cuzick J., Dowsett M., Pineda S., et al.. Prognostic value of a combined estrogen receptor, progesterone receptor, Ki-67, and human epidermal growth factor receptor 2 immunohistochemical score and comparison with the genomic health recurrence score in early breast cancer. J Clin Oncol. 2011; 29: 4273-4278.

17. Hugh J., Hanson J., Cheang M.C., et al.. Breast cancer subtypes and response to docetaxel in node-positive breast cancer: use of an Immunohistochemical definition in the BCIRG 001 Trial. J Clin Oncol. 2009; 27: 1168-1176.
18. Cheang M.C., Chia S.K., Voduc D., et al. Ki67 Index, HER2 Status, and prognosis of patients with luminal B breast Cancer. J Natl Cancer Inst. 2009; 101: 736-750.

19. Gayathri Nagaraj, Ma C.X. Adjuvant Chemotherapy Decisions in Clinical Practice for Early-Stage Node-Negative, Estrogen Receptor-Positive, HER2-Negative Breast Cancer: Challenges and Considerations. JNCCN. 2013; 11(3): 246-251.

20. Волченко А.А., Чиссов В.И., Зикиряходжаев А.Д. Выбор реконструктивно-пластических операций при комплексном лечении больных раком молочной железы. Онкология. Журнал им. П.А. Герцена. 2014; № 1: 3-9.

21. Bleyer A., Welch H.W. Effect of Three Decades of Screening Mammography on Breast-Cancer Incidence. N Engl J Med. 2012; 367: 1998- 2005.

22. Besluit van 16 juli 2001, houdende vaststelling van het besluit stralingsbescherming.12. 2. URL: http://www.sbng.nl (accessed 20.04.2015).

23. Elston C.W., Ellis I.O. Pathological prognostic factors in breast cancer. The value of histological grade in breast cancer: experience from a large study with long-term follow-up. Histopathology. 1991; 19: 403-410.

24. Cianfrocca M., Goldstein L.J. Prognostic and predictive factors in early-stage breast cancer. Oncologist. 2004; 9: 606-616.

25. Dowsett M., Lopez-Knowles E., Sidhu K., et al. Comparison of PAM50 risk of recurrence (ROR) score with Oncotype DX and IHC4 for predicting residual risk of RFS and distant-(D)RFS after endocrine therapy: a transATAC study. Cancer Res. 2011; 71(3): 4-5.

26. Dabbs D.J., Klein M.E., Mohsin S.K., et al. High false-negative rate of HER2 quantitative reverse transcription polymerase chain reaction of the Oncotype DX test: an independent quality assurance study. J Clin Oncol. 2011; 29: 4279-4285.

27. Baehner F.L., Achacoso N., Maddala T., et al. Human epidermal growth factor receptor 2 assessment in a case-control study: comparison of fluorescence in situ hybridization and quantitative reverse transcription polymerase chain reaction performed by central laboratories. J Clin Oncol. 2010; 28: 4300-4306.

28. Hammond M.E., Hayes D.F., Dowsett M. et al. American Society of Clinical Oncology/ College of American Pathologists guideline recommendations for immunohistochemical testing of estrogen and progesterone receptors in breast cancer. J Clin Oncol. 2010; 28(16): 2784-2795.

29. Hammond M.E., Hayes D.F., Wolff A.C. Clinical Notice for American Society of Clinical Oncology-College of American Pathologists guideline recommendations on ER/PgR and HER2 testing in breast cancer. J Clin Oncol. 2011; 29: e458.

30. Vaz-Luis I., Winer E.P., Lin N. U. Human epidermal growth factor receptor-2-positive breast cancer: does estrogen receptor status define two distinct subtypes? Annals of Oncology. 2013;.24: 283-291.

31. Paik S., Tang G., Shak S., et al. Gene expression and benefit of chemotherapy in women with node-negative, estrogen receptor-positive breast cancer. J Clin Oncol. 2006; 24: 37263734.

32. Carlson R.W., Allred D.C., Anderson B.O., et al. NCCN Clinical Practice Guidelines in Oncology: Breast Cancer. Version 3, 2012. URL: www.nccn.org (accessed 17.01.2013). 
33. Mook S., Schmidt M.K., Rutgers E.J., et al. Calibration and discriminatory accuracy of prognosis calculation for breast cancer with the online Adjuvant! program: a hospital-based retrospective cohort study. Lancet Oncol. 2009; 10: 1070-1076.

34. Caruso F., Ferrara M., Castiglione G., Trombetta G., De Meo L., Catanuto G., Carillio G. Nipple sparing subcutaneous mastectomy: sixty-six months follow-up. Eur J Surg Oncol.2006; 32(9): P.937.

35. Sacchini V.S., de Alcantara Filho P., Capko D., Barry J.M., Morrow M. Nipple-sparing mastectomy for breast cancer and risk-reducing surgery: the Memorial Sloan-Kettering Cancer Center experience. Ann Surg Oncol. 2011; 18(11): 3117-22.

36. Komorowski A.L., Zanini V., Regolo L., Carolei A., Wysocki W.M., Costa A. Necrotic complications after nipple- and areolasparing mastectomy.World J Surg. 2006; 30(8): 1410.

37. Galimberti V., Cole B.F., Zurrida S.. et al. Axillary dissection versus no axillary dissection in patients with sentinel-node micrometastases (IBCSG 23-01): a phase 3 randomised controlled trial. Lancet Oncol. 2013; 14: 297-305.

38. Giuliano A.E., Hunt K.K., Ballman K.V.. et al. Axillary dissection versus no axillary dissection in women with invasive breast cancer and sentinel node metastasis. JAMA 2011; 305: 569575.

39. Voogd A.C., van Oost F.J., Rutgers E.J., et al. Long term prognosis of patients with local recurrence after conservative surgery and radiotherapy for early breast cancer. Eur J cancer. 2005; 41: 2637-2644.

40. Hughes J.H., Mason M.C., Gray R.J., et al. A Multi-site validation trial of radioactive seed localization as an alternative to wire localization. Breast J. 2008; 14: 153-157.

41. Lovrics P.J., Goldsmith C.H., Hodgson N., et al. A multicentered, randomized, controlled trial comparing radio guided seed localization to standard wire localization for nonpalpable invasive and in situ breast carcinomas. Ann Surg Oncol. 2011; 18: 3407-3414.

42. Centraal BegeleidingsOrgaan ( $\mathrm{CBO}$ ). Fysieke en cognitieve effecten, voorlichting en psychosociale zorg. In: Richtlijn mammacarcinoom 2008. P. 222-224.

43. Krekel N.M., Zonderhuis B.M., Scheurs H.W.H., et al. Ultrasoundguided breast-sparing surgery to improve cosmetic outcomes and quality of life. A prospective multicentre randomized controlled clinical trial comparing ultrasound-guided surgery to traditional palpation guided surgery (COBALT trial). BMC Surg. 2011; 16: 11-18

44. Pleijhuis R.G., Langhout G.C., Helfrich W., et al. Near-infrared fluorescence (NIRF) imaging in breast-conserving surgery: assessing intraoperative techniques in tissue-simulating breast phantoms. Eur J Surg oncol.. 2011;.37: 32-39.

45. Espinosa-Bravo M., Sao Aviles A., Esqueva A., et al. Breast conservative surgery after neoadjuvant chemotherapy in breast cancer patients: comparison of two tumor localization methods. Eur J Surg Oncol. 2011; 37: 1038-1043.

46. Krekel N.M., Zonderhuis B.M., Stockmann H.B., et al. A comparison of three methods for nonpalpable breast cancer excision. Eur J Surg Oncol. 2011; 37: 109-115.
47. Weissenbacher T.M., Zschage M., Janni W., et al. Multicentric and multifocal versus unifocal breast cancer: is the tumornode-metastasis classification justified? Breast Cancer Res Treat. 2010; 122: 27-34.

48. Bauman L., Barth R.J., Rosenkranz K.M.. Breast conservation in women with multifocal-multicentric breast cancer: is it feasible? Ann Surg Oncol. 2010; 17: 325-329.

49. Yerushalmi R., Tyldesley S., Woods R., et al. Is breast conserving therapy a safe option for patients with tumor multicentricity and multifocality. Ann Oncol. 2012; 23: 876-881.

50. Massarweh S., Osborne C.K., Jiang S., et al. Mechanisms of tumor regression and resistance to estrogen deprivation and fulvestrant in a model of estrogen receptor-positive, HER-2/ neu-positive breast cancer. Cancer Res. 2006; 66: 8266-8273.

51. Guarneri V., Frassoldati A., Bottini A., et al. Final results of a phase II randomized trial of neoadjuvant anthracyclinetaxane chemotherapy plus lapatinib, trastuzumab, or both in HER2-positive breast cancer (CHER-LOB trial) [abstract]. J Clin Oncol. 2011; 29: a507.

52. Baselga J., Bradbury I., Eidtmann H., et al. Lapatinib with trastuzumab for HER2- positive early breast cancer (NeoALTTO): a randomised, open-label, multicentre, phase 3 trial. Lancet.2012; 379: 633-640.

53. Shou J., Massarweh S., Osborne C.K., et al. Mechanisms of tamoxifen resistance: increased estrogen receptor-HER2/neu cross-talk in ER/HER2-positive breast cancer. J Natl Cancer Inst. 2004; 96: 926-935.

54. Viale G., Regan M.M., Maiorano E., et al.. Prognostic and predictive value of centrally reviewed expression of estrogen and progesterone receptors in a randomized trial comparing letrozole and tamoxifen adjuvant therapy for postmenopausal early breast cancer: BIG 1-98. J Clin Oncol. 2007; 25: 38463852.

55. Slamon D., Eiermann W., Robert N., et al. Adjuvant trastuzumab in HER2-positive breast cancer. N Engl J Med. 2011; 365: 12731283.

56. Perez E.A., Romond E.H., Suman V.J., et al. Four-year follow-up of trastuzumab plus adjuvant chemotherapy for operable human epidermal growth factor receptor 2- positive breast cancer: joint analysis of data from NCCTG N9831 and NSABP B- 31. J Clin Oncol. 2011; 29: 3366-3373.

57. Albain K., Barlow W.E., Shak S., et al. Prognostic and predictive value of the 21-gene recurrence score assay in postmenopausal women with node-positive,oestrogen-receptor-positive breast cancer on chemotherapy: a retrospective analysis of a randomised trial. Lancet Oncology. 2010; 11(1): 55-65.

58. Hornberger J., Chien R. Meta-analysis of the decision impact of the 21-gene breast cancer Recurrence Score in clinical practice. In Poster Presented at the St Gallen International Breast Cancer Conference, St Gallen, Switzerland, March. 2011; Abstract: 201. 
Информация об авторах:

1. Трошенков Евгений Алексеевич, к.м.н., ведущий хирург отделения общей онкологии МНИОИ им. П.А. Герцена - филиал ФГБУ «НМИРЦ» Минздрава России

2. Костин Андрей Александрович, д.м.н., профессор, первый заместитель генерального директор ФГБУ “НМИРЦ» Минздрава России, заведующий кафедрой урологии, онкологии и радиологии ФПК МР РУДН
Information about authors:

1. Troshenkov Evgeniy Alexeevich - PhD, leading surgeon of department of general oncology of P. Hertsen MORI

2. Kostin Andrey Alexandrovich - MD., professor, vice director of NMRRC

Оформление ссылки для цитирования статьи:

Трошенков Е.А., Костин А.А. Проблемы диагностики и лечения первично операбельного рака молочной железы.

Исследования и практика в медицине. 2015; 2(2): 35-43. DOI: 10.17709/2409-2231-2015-2-2-35-43

Troshenkov E.A., Kostin A.A. Problems of diagnosis and treatment of primary operable breast cancer. Issled. prakt. Med. 2015; 2(2): 35-43. DOI:10.17709/2409-2231-2015-2-2-35-43

Конфликт интересов. Все авторы сообщают об отсутствии конфликта интересов.

Conflict of interest. All authors report no conflict of interest. 